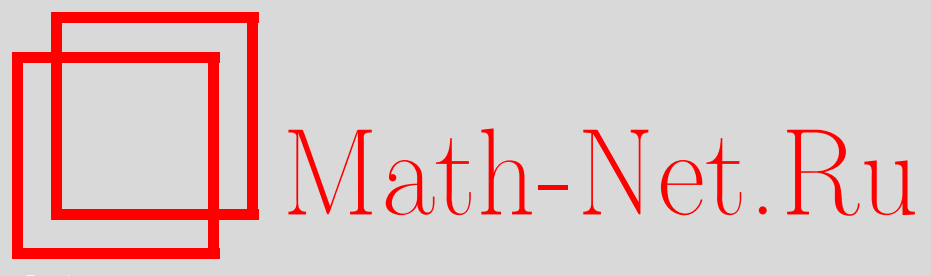

А. В. Петухов, Критерий аффинности фактора алгебраической группы по одномерной подгруппе, УМН, 2007, том 62, выпуск 5, 161-162

DOI: https://doi.org/10.4213/rm7596

Использование Общероссийского математического портала Math-Net.Ru подразумевает, что вы прочитали и согласны с пользовательским соглашением http://www . mathnet.ru/rus/agreement

Параметры загрузки:

IP : 54.224 .187 .69

26 апреля 2023 г., $07: 39: 52$

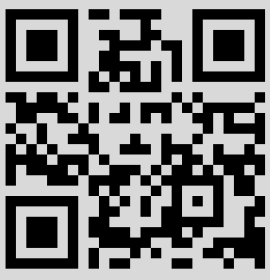




\title{
Критерий аффинности фактора алгебраической группы по одномерной подгруппе
}

\author{
А. В. Петухов
}

В работе доказано, что однородное пространство аффинной алгебраической группы $G$ по одномерной унипотентной подгруппе $H$ аффинно тогда и только тогда, когда эта подгруппа не содержится ни в какой редуктивной подгруппе группы $G$.

Пусть $G$ - аффинная алгебраическая группа над алгебраически замкнутым полем характеристики нуль, а $H$ - ее замкнутая подгруппа. Хорошо известно, что однородное пространство $G / H$ имеет каноническую структуру квазипроективного $G$-многообразия, однако остается открытым вопрос о том, для каких пар $(G, H)$ оно аффинно. Ответ в случае редуктивной $G$ был найден Й. Мацусимой [1] и выглядит так: $G / H$ аффинно тогда и только тогда, когда $H$ редуктивна. В работе [2] доказано, что $G / H$ аффинно тогда и только тогда, когда $G / H^{\mathrm{u}}$ аффинно, где $H^{\mathrm{u}}$ - унипотентный радикал $H$. Более того, легко показать, что если $G / H^{\mathrm{u}}$ аффинно, то и $G / F$ аффинно для любой унипотентной подгруппы $F \subset H^{\mathrm{u}}$. Цель настоящей работы определить, когда однородное пространство по одномерной унипотентной группе $H$ аффинно.

Теорема 1. Однородное пространство $G / H$ аффинной алгебраической группъ $G$ по одномерной унипотентной подгруппе $H$ аффинно тогда и толъко тогда, когда $H$ не содержится ни в какой редуктивной подгруппе группы $G$.

Пусть $A \supset B \supset C$ - алгебраические группы.

Лемма 1 [3; с. 45]. Если $A / B$ и $B / C$ аффинны, то и $A / C$ аффинно.

Лемма 2. Если $A / B$ аффинно, то $A / C$ аффинно тогда и только тогда, когда В/C аффинно.

ДоказАтельство. Если $B / C$ аффинно, то и $A / C$ аффинно по лемме 1 . Если же $A / C$ аффинно, то $B / C$ тоже аффинно, так как является образом замкнутого $C$-инвариантного многообразия $B$ при геометрическом факторе $A \rightarrow A / C$.

ОпредЕлЕниЕ 1 . Пусть $H$ - алгебраическая подгруппа алгебраической группы $G$. Сечением для действия $H: G$ называется подмногообразие, пересекающееся с каждой орбитой ровно один раз и с каждой компонентой связности группы $G$ по неприводимому подмногообразию.

ОСНовная ЛЕмма. Пусть $G=L \curlywedge_{\rho} V$, где $L$ - редуктивная группа с представлением $\rho: L \rightarrow \mathrm{GL}(V)$. Пусть $h$ - унипотентный элемент группы $G$, а $H$ - минимальная алгебрачческая подгруппа, его содержащая. Тогда либо существует элемент $h^{\prime} \in L$, сопряженный $h$, либо существует сечение для действия $H: G$.

ДоказАтельство. Представим $h$ в виде $l \cdot v, l \in L, v \in V$. Возникает два случая.

1) Если $v \in \operatorname{Im}(\rho(l)-\operatorname{Id})$, то существует $f \in V$ такой, что $h^{\prime}=f^{-1} h f \in L$.

2) Если $v \notin \operatorname{Im}(\rho(l)-\mathrm{Id})$, то существует гиперплоскость $V^{\prime} \subset V$, содержащая $\operatorname{Im}(\rho(l)-\mathrm{Id})$ и такая что $V=\langle v\rangle \oplus V^{\prime}$. Покажем, что $K=L V^{\prime}$ (прообраз $V^{\prime}$ при отображении $G \rightarrow L \backslash G)$ - сечение действия $H: G$. Так как $\rho(l)$ сохраняет $\operatorname{Im}(\rho(l)-\operatorname{Id})$, а на факторе $V / \operatorname{Im}(\rho(l)-\operatorname{Id})$ действует тождественно, получаем действие $H: V / \operatorname{Im}(\rho(l)-\mathrm{Id})$ параллельными переносами. У него, очевидно, существует сечение, являющееся линейным подпространством; его прообраз мы и обозначим $V^{\prime}$. Так как $H$ действует на $V / V^{\prime}$ свободно и транзитивно, то прообраз нуля при отображении $G \rightarrow L \backslash G \rightarrow(L \backslash G) / V^{\prime}$ будет сечением. 
ДокАЗАТЕльство теоРемы 1. Очевидно, что если существует редуктивная подгруппа $S$ группы $G$, включающая $H$, то, так как $S / H$ неаффинно (критерий Мацусимы), $G / H$ тоже неаффинно (лемма 2 ). Осталось доказать, что если такой подгруппы $S$ не существует, то фактор аффинен.

Доказательство будем вести индукцией по размерности унипотентного радикала $G^{\mathrm{u}}$ группы $G$. Если $\operatorname{dim} G^{\mathrm{u}}=0$, то утверждение очевидно. Пусть теперь $\operatorname{dim} G^{\mathrm{u}}>0$. Пусть $L$ - максимальная редуктивная подгруппа группы $G$. Рассмотрим произвольный неединичный элемент $h$ из $H$ и его представление $h=l \cdot r$, где $l \in L$, a $r \in G^{\mathrm{u}}$. Заметим, что $L$ действует сопряжением на векторном пространстве $V=$ $G^{\mathrm{u}} /\left(G^{\mathrm{u}}, G^{\mathrm{u}}\right)$ и, в силу основной леммы, либо $h$ сопряжен какому-то элементу $h^{\prime}$, лежащему в $L \curlywedge\left(G^{\mathrm{u}}, G^{\mathrm{u}}\right)$, либо существует сечение для действия $H$ на $L \curlywedge\left(G^{\mathrm{u}} /\left(G^{\mathrm{u}}, G^{\mathrm{u}}\right)\right)$ и, следовательно, на $G$. Во втором случае ограничение отображения факторизации $G \rightarrow G / H$ на сечение биективно и фактор гладок, а отсюда следует, что он аффинен. Вернемся теперь к первому случаю. Пусть $H^{\prime}$ - минимальная алгебраическая подгруппа, содержащая элемент $h^{\prime}$. Тогда $G / H \cong G / H^{\prime}$, однако $\left(L \curlywedge\left(G^{\mathrm{u}} /\left(G^{\mathrm{u}}, G^{\mathrm{u}}\right)\right)\right) / L$ аффинно, а $\left(L \curlywedge\left(G^{\mathrm{u}}, G^{\mathrm{u}}\right)\right) / H^{\prime}$ аффинно по предположению индукции. По лемме 2 отсюда следует, что и $G / H$ аффинно. Доказательство теоремы 1 завершено.

Приведем пример аффинного однородного пространства по одномерной подгруппе, не лежащей в радикале. Таковым будет фактор группы аффинных преобразований плоскости по подгруппе, порожденной векторным полем $y \frac{\partial}{\partial x}+\frac{\partial}{\partial y}$.

Таким образом, в случае одномерной унипотентной $H$ однородное пространство $G / H$ аффинно тогда и только тогда, когда $H$ не пересекается ни с какой редуктивной подгруппой из $G$. Можно показать, что это условие является необходимым для аффинности фактора по произвольной унипотентной группе, но не является достаточным: соответствующий контрпример для двумерной унипотентной подгруппы приведен в [4].

Автор выражает огромную благодарность И. В. Аржанцеву и Э. Б. Винбергу, без помощи и поддержки которых эта работа так никогда бы и не была опубликована.

\section{Список литературы}

[1] Y. Matsushima, Nagoya Math. J., 16 (1960), 205-218. [2] Y. Matsushima, A. Marimoto, Bull. Soc. Math. France, 88 (1960), 137-155. [3] F. D. Grosshans, Algebraic homogeneous spaces and invariant theory, Lecture Notes in Math., 1673, Springer-Verlag, Berlin, 1997. [4] J. Winkelmann, Math. Ann., 286:1-3 (1990), 593-612.

\section{А. В. Петухов (А. V. Petukhov)}

Московский государственный университет им. М. В. Ломоносова

E-mail: petushok@mail.ru
Представлено Э. Б. Винбергом Принято редколлегией 31.07 .2007 